\title{
Quasistatic to inertial transition in granular materials and the role of fluctuations
}

\author{
Johan Gaume, Guillaume Chambon, ${ }^{*}$ and Mohamed Naaim \\ Cemagref, UR ETGR, 2 rue de la Papeterie, FR-38402 St. Martin d' Hères Cedex, France \\ (Received 16 July 2010; revised manuscript received 30 September 2011; published 11 November 2011)
}

\begin{abstract}
On the basis of discrete element numerical simulations of a Couette cell, we revisit the rheology of granular materials in the quasistatic and inertial regimes, and discuss the origin of the transition between these two regimes. We show that quasistatic zones are the seat of a creep process whose rate is directly related to the existence and magnitude of velocity fluctuations. The mechanical behavior in the quasistatic regime is characterized by a three-variable constitutive law relating the friction coefficient (normalized stress), the inertial number (normalized shear rate), and the normalized velocity fluctuations. Importantly, this constitutive law appears to remain also valid in the inertial regime, where it can account for the one-to-one relationship observed between the friction coefficient and the inertial number. The abrupt transition between the quasistatic and inertial regimes is then related to the mode of production of the fluctuations within the material, from nonlocal and artificially sustained by the boundary conditions in the quasistatic regime, to purely local and self-sustained in the inertial regime. This quasistatic-to-inertial transition occurs at a critical inertial number or, equivalently, at a critical level of fluctuations.
\end{abstract}

DOI: 10.1103/PhysRevE.84.051304

PACS number(s): 83.80.Fg, 47.57.Gc

\section{INTRODUCTION}

One of the most fascinating properties of granular materials is their ability to either sustain stresses as solids, or to flow as fluids, depending on the applied solicitation. It has been shown in several studies [1-3] that, at any given point within the material, this solid-to-fluid transition is primarily controlled by the local value of the inertial number $I=\dot{\gamma} d(\rho / P)^{0.5}$. This number represents the ratio between a microscopic inertial timescale $d(\rho / P)^{0.5}$ ( $P$ being the pressure, $d$ the grain diameter, and $\rho$ the grain density) and the macroscopic time scale $\dot{\gamma}^{-1}$ associated with the shear rate $\dot{\gamma}$. Fluidlike behavior is obtained for large enough values of $I$ and corresponds to the so-called inertial regime. In this regime, the constitutive law of the material is characterized by a one-to-one relationship, of the viscoplastic type, between the friction coefficient $\mu=\tau / P$ and the inertial number $I[4,5]$. For low values of $I$, on the contrary, the $\mu(I)$ relationship loses its validity and solidlike behavior is recovered. In this so-called quasistatic regime, and in agreement with the plastic constitutive laws classically used in soil mechanics for $I \rightarrow 0$ [6], the mechanical behavior is generally described as becoming rate independent and characterized by a constant friction coefficient $\mu_{s}$ (critical state theory).

The transition between the inertial and quasistatic regimes typically occurs for values of $I$ in the range $10^{-3}-10^{-2}$. In detail, however, the reported $I$ value at the transition appears to vary between existing studies, and possibly depends on system size $[1,2]$. Furthermore, from these studies, it is still not clear whether this transition occurs sharply at a given value of $I$, or progressively as $I \rightarrow 0$. More generally, the real nature of the quasistatic-to-inertial transition in granular materials, and the physical processes involved, still remain largely unknown. Recently, the mechanical behavior in the quasistatic regime has been shown to be significantly more complex than described by classical soil mechanics. In particular, continuous

\footnotetext{
*guillaume.chambon@cemagref.fr
}

creep in quasistatic zones, incompatible with a supposedly rate-independent mechanical behavior, has been reported in several configurations (such as free-surface flows and Couette cells) $[3,7,8]$. Several studies have also evidenced the existence, in the quasistatic regime, of strong and intermittent fluctuations characterized by collective particle motions with large correlation lengths (nonlocal processes) [9-13]. Yet, and although it is reasonable to think these fluctuations may play an important role in the macroscopic rheology of the material $[12,14,15]$, the link between fluctuations and creep has never been formally proved. Similarly, although the existence of creep in the quasistatic regime could lead one to think that the transition toward the "true" solid behavior is in fact progressive, this issue, as well as the potential connection between the quasistatic creep and the viscoplastic rheology in the inertial regime, remains to be properly addressed.

The objective of this paper is precisely to explore the links between creep, fluctuations, and viscoplastic rheology in order to propose a more consistent description of the quasistatic-to-inertial transition in granular materials. We will prove that the creep is effectively related to the existence of fluctuations within the sample, and that a three-variable constitutive law between shear rate, friction coefficient, and fluctuation level can be formulated in the quasistatic regime. In addition, we will show that this constitutive law remains valid in the inertial regime, where it can account with good accuracy for the viscoplastic behavior observed, thus opening the way toward a unified treatment of both quasistatic and inertial regimes.

Our work is based on numerical simulations using the discrete element method (DEM) [16]. This method allows us to perform veritable numerical experiments on granular materials. The configuration simulated is a Couette cell (or annular shear cell), which presents the specificity of placing locally the tested sample in a state of simple shear, but with a shear stress $\sigma_{r \theta}$ that decreases from the inner wall to the outer sample boundary according to $\sigma_{r \theta}(r)=\sigma_{r \theta}\left(R_{i}\right) R_{i}{ }^{2} / r^{2}$ (where $R_{i}$ is the inner cylinder radius and $r$ the radial position of the point considered) $[3,10,15,17-19]$. This setting is thus 
particularly well suited to studying the quasistatic-to-inertial transition, since coexistence between quasistatic and inertial zones can be observed within the same sample. It is important to mention that our simulations are very similar to those reported in [3]. This latter study actually presents a complete overview of the mechanical response of a granular sample placed in a Couette cell, which we shall obviously not reproduce here. In the present paper, we take one step forward and, building from the results of [3], our analysis of the simulation data is specifically focused on insights concerning sample rheology and the quasistatic-to-inertial transition.

This paper is organized as follows. Section II presents the simulated system and defines the mechanical quantities considered. In Sec. III, after having evidenced the existence of the quasistatic-to-inertial transition, we revisit the rheological properties of the inertial regime and propose a new approach to the rheology of the quasistatic regime. In Sec. IV, an empirical three-variable constitutive law valid in both the quasistatic and the inertial regimes is derived. Lastly, conclusions regarding the physical nature of these two regimes and of the transition between them are discussed in Sec. V.

\section{SIMULATED SYSTEM}

The discrete element simulations were performed using the commercial software PFC2D (by Itasca) which implements the original soft-contact algorithm described in [20]. The simulated Couette cell is two dimensional (Fig. 1), with inner and outer cylinder radii $R_{i}=0.4 \mathrm{~m}$ and $R_{e}=0.6 \mathrm{~m}$. The granular samples are composed of about 7000 circular particles of average diameter $d=4.7 \mathrm{~mm}$ [thus $\left(R_{e}-R_{i}\right) / d \approx 43$ ], with a grain size distribution polydispersity of $\pm 30 \%$ (diameters ranging from 3 to $6 \mathrm{~mm}$ ) in order to prevent crystallization. Shear is applied by rotation of the inner cylinder at an imposed rotation velocity $\Omega$ which was varied between 0.05 and $20 \mathrm{rad} \mathrm{s}^{-1}$. The outer cylinder is fixed, but consists of a flexible membrane through which a constant radial pressure $P=10 \mathrm{kPa}$ is imposed onto the sample. This setting is preferable to a rigid wall, in order to accommodate the density variations undergone by the granular material during shear.

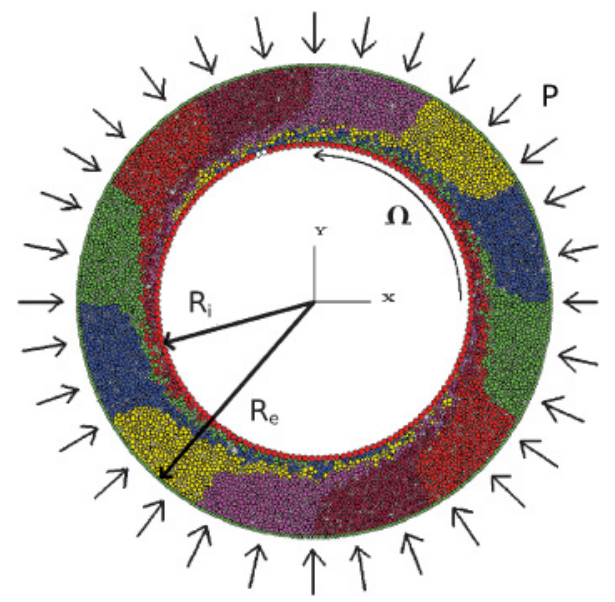

FIG. 1. (Color online) Simulated shear cell. Zones of different colors within the sample illustrate the shear deformation.
TABLE I. Mechanical parameters used in the simulations. $k_{n}$ : normal contact stiffness; $k_{t}$ : tangential contact stiffness; $\mu_{g}:$ intergranular friction; $e$ : normal restitution coefficient; $\rho$ : particle density.

\begin{tabular}{ccccc}
\hline \hline$k_{n} / P$ & $k_{t} / k_{n}$ & $\mu_{g}$ & $e$ & $\rho$ \\
\hline $10^{4}$ & 0.5 & 0.5 & $0.1,0.9$ & $300 \mathrm{~kg} \mathrm{~m}^{-3}$ \\
\hline \hline
\end{tabular}

Both boundaries are constituted by grains of diameter $d$ to represent wall roughness.

The interparticle contact laws used in the simulations are classical [16]. The normal force is the sum of a linearelastic and of a viscous contribution (spring-dashpot model), and the tangential force is linear-elastic with a Coulombian friction threshold. The corresponding mechanical parameters are summarized in Table I. Let us mention that the value of the normal stiffness $k_{n}$ was chosen in order to keep low normal interpenetrations $\delta$ at contacts, $\delta / d \ll 10^{-3}$, i.e., to work in the quasirigid grain limit $[2,6]$. Concerning the normal restitution coefficient $e$, we checked that the results presented below, and more generally all the macroscopic mechanical quantities obtained from the simulations, are actually independent of this parameter (in the range $0.1-0.9$ ), in agreement with previous studies [2].

One of the main interests of DEM simulations is that mechanical quantities such as stresses, shear rates, etc., can be computed at each material point within the sample. Hence, the rheological behavior of the material can be explored locally, regardless of the spatial heterogeneities possibly displayed by these mechanical quantities. In our case, the shear rate $\dot{\gamma}$ is obtained from the orthoradial velocity profile $v(r)$ according to $\dot{\gamma}=r[d(v / r) / d r]$. The stress tensor is derived using the classical Love homogenization formula [21]. In the following, only mechanical responses obtained in steady state will be considered, disregarding the transients that occur

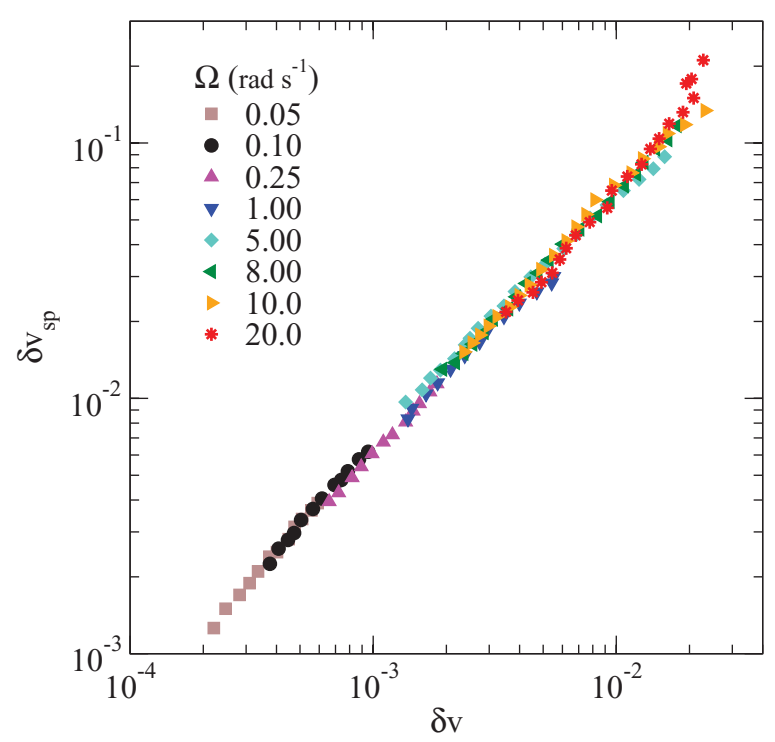

FIG. 2. (Color online) Correlation between the spatiotemporal velocity fluctuations $\delta v_{\text {sp }}$ and the temporal velocity fluctuations $\delta v$ in the simulated samples. The different symbols refer to the imposed values of inner cylinder rotation velocity $\Omega$. 

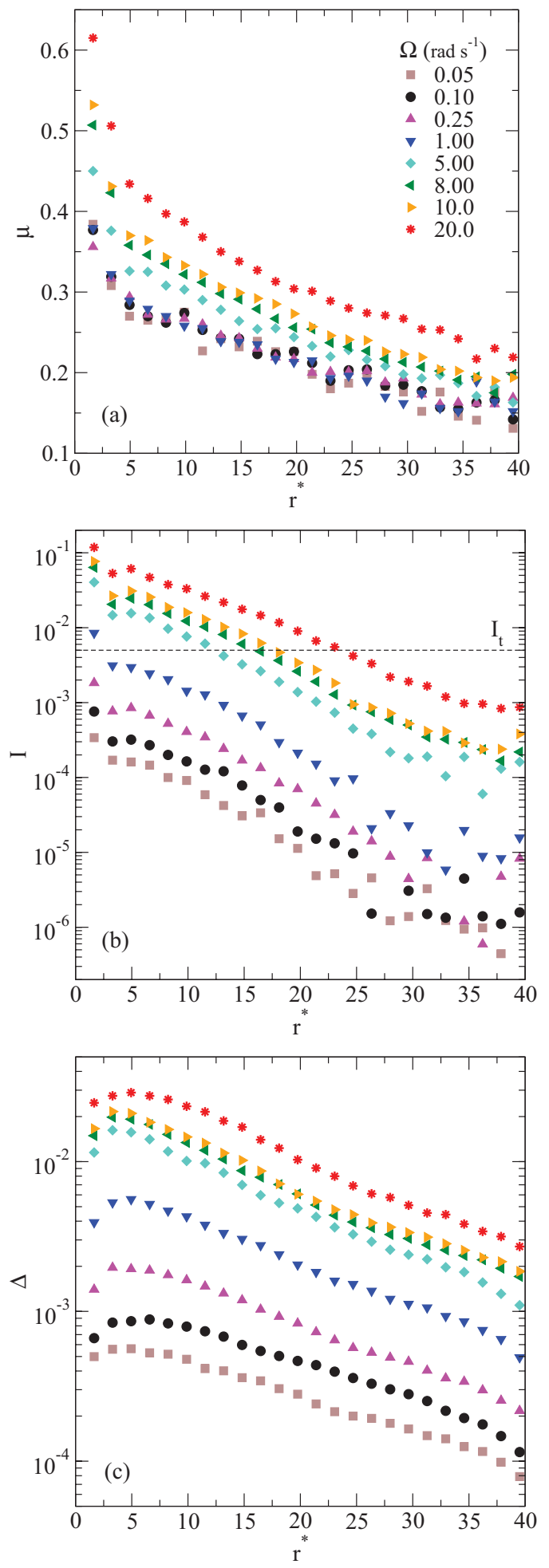

FIG. 3. (Color online) Radial profiles of (a) friction coefficient $\mu$, (b) inertial number $I$, and (c) fluctuation number $\Delta$ for the different imposed values of inner cylinder rotation velocity $\Omega$. The profiles are plotted as a function of the reduced radius $r^{*}=\left(r-R_{i}\right) / d$.

at the initiation of shear. Acknowledging this steady-state condition and the cylindrical symmetry of the system, all the mechanical quantities computed are subjected to a double, spatiotemporal averaging procedure $\langle\cdot\rangle_{\theta, t}$ over annuli having a thickness of $1.6 d$ and over time windows that are sufficiently long to integrate both individual and correlated particle motions.

In addition to average quantities, we will also consider the orthoradial velocity fluctuations $\delta v=\left(\left\langle\langle v\rangle_{\theta}{ }^{2}\right\rangle_{t}-\langle v\rangle_{\theta, t}{ }^{2}\right)^{1 / 2}$. Note that defined as such, the quantity $\delta v$ only accounts for the temporal fluctuations of the spatially averaged velocity $\langle v\rangle_{\theta}$. We chose this fluctuation measure by analogy with the common practice in fluid turbulence. In addition, as shown in Fig. 2, we observed that the spatiotemporal velocity fluctuations defined as $\delta v_{\mathrm{sp}}=\left(\left\langle v^{2}\right\rangle_{\theta, t}-\langle v\rangle_{\theta, t}{ }^{2}\right)^{1 / 2}$ appear strongly correlated to the temporal fluctuations $\delta v$ in our system. Hence, and although this result would deserve further discussion beyond the scope of this paper, we argue that the quantity $\delta v$ can actually be considered as a good proxy for all types of fluctuations within our samples.

Compared to the simulations described in [3], the only notable specificity of our study lies in the consideration of complete annular samples, instead of orthoradial periodic boundary conditions. As a validation of our work, we checked that the global response of the samples observed in our simulations, such as the evolutions with $r$ and $\Omega$ of the velocity, density, and stresses, fully agrees with the results presented in [3]. As already mentioned, the reader is thus referred to this previous study to get an overall view of the mechanical behavior of a granular sample in a Couette cell. In what follows, we only focus on the variables relevant to describing the macroscopic rheology of the granular material, namely, the inertial number $I$ (dimensionless shear rate), the friction coefficient $\mu$ (dimensionless shear stress), and a dimensionless measure of the velocity fluctuations $\Delta$ defined as $\Delta=\delta v(\rho / P)^{0.5}$.

The radial profiles within the sheared samples of the quantities $I, \mu$, and $\Delta$ are shown in Fig. 3. The observed decrease of the friction coefficient $\mu$ with $r$ is fully explainable by the geometrical heterogeneity of the shear stress inside the Couette cell: $\mu \propto\left(r / R_{i}\right)^{-2}$ (the pressure being constant in the sample; see [3]). In parallel, both the inertial number $I$ and the velocity fluctuations $\Delta$ also decrease with $r$. These two quantities display roughly exponential trends, with characteristic lengths that remain quasiconstant for all tested values of the rotation velocity $\Omega$. (In detail, however, the localization width, i.e., the characteristic length associated with the exponential decrease of $I$, may show a slight increase with $\Omega$; see [3].)

\section{GRANULAR RHEOLOGY}

\section{A. A marked quasistatic-to-inertial transition}

The mechanical behavior of the tested material is highlighted when representing directly the friction coefficient $\mu$ or the fluctuation number $\Delta$ as a function of the inertial number $I$ for all locations within the sample and all rotation velocities $\Omega$ (Fig. 4). As already noted in [3], the plot of $\mu$ versus $I$ [Fig. 4(a)] clearly evinces the existence of a marked rheological transition at a given value of $I, I_{t} \approx 5 \times 10^{-3}$ in our case. For $I>I_{t}$, all the obtained data points collapse on a master curve and, therefore, define a single $\mu(I)$ relationship regardless of the values of rotation velocity $\Omega$ and radius $r$. For $I<I_{t}$, on the contrary, there appears to be no one-to-one relationship 

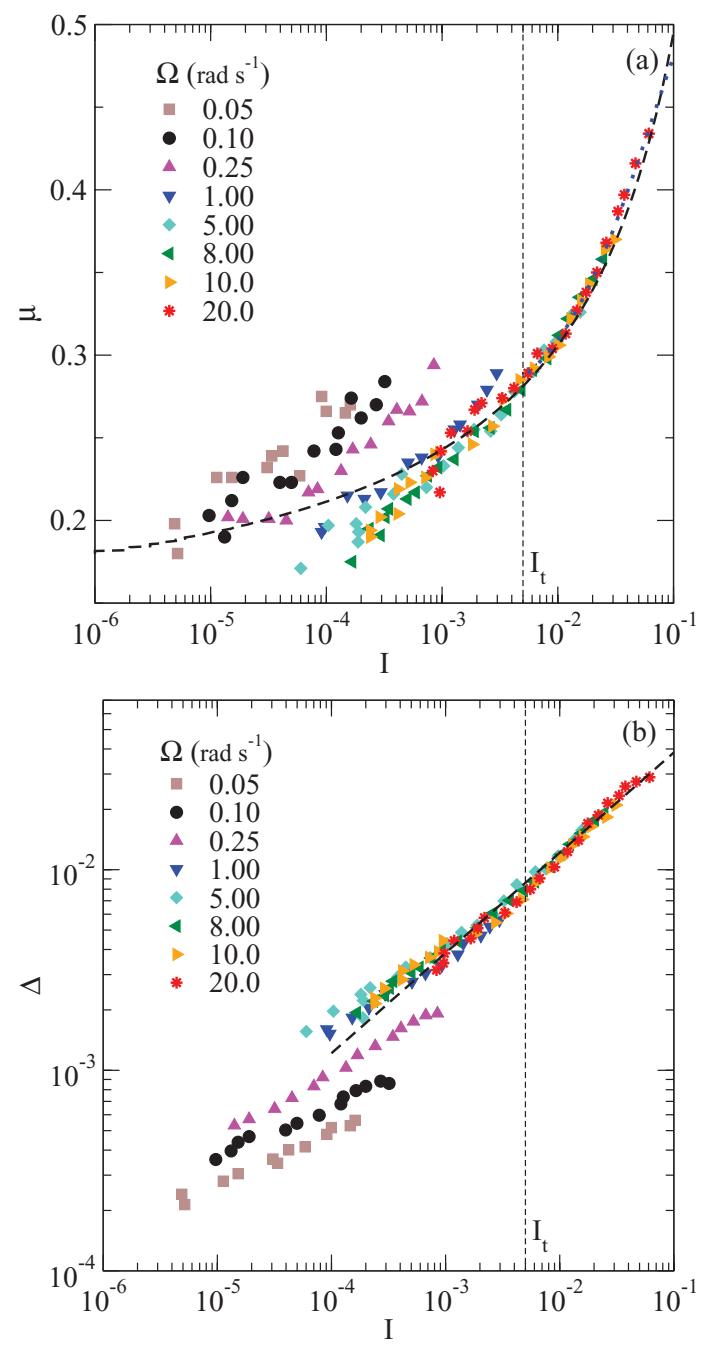

FIG. 4. (Color online) (a) Friction coefficient $\mu$ as a function of inertial number $I$. The different symbols refer to the imposed values of inner cylinder rotation velocity $\Omega$. The blue dotted curve represents Eq. (1), while the black dashed one corresponds to Eq. (6). (b) Fluctuation number $\Delta$ as a function of inertial number $I$. The dashed line represents Eq. (2).

between $\mu$ and $I$. Interestingly, this transition at $I=I_{t}$ is also clearly visible on the evolution of the fluctuation number $\Delta$ with $I$ [Fig. 4(b)]. Similarly, a one-to-one relationship $\Delta(I)$ is obtained for $I>I_{t}$, while no such relationship exists for $I<I_{t}$.

Following [3], we identify this transition observed at $I=I_{t}$ with the quasistatic-to-inertial transition. The data points corresponding to the inertial $\left(I>I_{t}\right)$ and to the quasistatic $\left(I<I_{t}\right)$ regime will now be examined independently, in order to exhibit the rheological properties of each of these regimes. Let us recall that due to the decrease of the inertial number $I$ with $r$, zones lying in the inertial and in the quasistatic regime may simultaneously coexist within our samples. In what follows, these cases of coexistence between inertial and quasistatic zones will prove to be particularly informative in terms of rheological behavior. In detail, Fig. 3(b) shows that such a coexistence is actually observed only above a particular value of the rotation velocity, $\Omega_{t} \approx 1 \mathrm{rad} \mathrm{s}^{-1}$. For $\Omega>\Omega_{t}$,
$I>I_{t}$ at the sample's inner boundary and an inertial zone thus develops around the inner cylinder, surrounded by a quasistatic zone outside. The thickness of the inertial zone progressively decreases with $\Omega$, and vanishes at $\Omega_{t}$. For $\Omega<\Omega_{t}$, the whole sample lies in the quasistatic regime. Note that a quasistatic zone, either alone or in coexistence with an inertial zone, was always present in all our simulations.

\section{B. Inertial regime}

The rheological behavior observed for $I>I_{t}$ is fully consistent with the results obtained in previous studies dedicated to the inertial regime of granular materials $[1,2]$. In particular, the $\mu(I)$ relationship in Fig. 4(a) can be well fitted by the empirical expression proposed in [4]:

$$
\mu=\mu_{s}+\frac{\mu_{l}-\mu_{s}}{I_{0} / I+1}
$$

with parameters on the same order as those obtained from experimental data $\left(\mu_{s}=0.26, \mu_{l}=0.62, I_{0}=0.07\right)$. Note that a simpler, alternative expression to describe this $\mu(I)$ relationship will be proposed hereinafter. Independent of the particular fitting law used, the existence of such a one-to-one relationship between the friction coefficient $\mu$ and the inertial number $I$ indicates that the inertial regime is characterized by a rate-dependent rheological behavior similar to that of a complex fluid. As a macroscopic signature of this ratedependent behavior, Fig. 3(a) shows that as soon an inertial zone exists around the inner cylinder, the friction coefficient profile in the sample (and thus the global torque on the inner cylinder) increases with the rotation velocity $\Omega$.

In parallel, and also in good agreement with previous studies [1,2], the relationship between the fluctuation and inertial numbers $\Delta$ and $I$ observed for $I>I_{t}$ is well fitted by a power law with an exponent of 0.5 [Fig. 4(b)]:

$$
\Delta=C_{I} I^{1 / 2} \text {, }
$$

where $C_{I} \approx 0.12$. The existence of this one-to-one relationship $\Delta(I)$ can be interpreted as the fluctuations being created locally by the granular agitation resulting from the shear rate [2,15]. Actually, as will be discussed later, we propose that it is precisely this property of locality for the fluctuations that constitutes the "intrinsic" definition of the inertial regime.

\section{Quasistatic regime}

In contrast to the inertial regime, the quasistatic regime is characterized by the absence of one-to-one relationships between $\mu$ and $I$ and between $\Delta$ and $I$. We also observe in Fig. 3(a) that the friction coefficient radial profiles are independent of the rotation velocity $\Omega$ for $\Omega<\Omega_{t}$, i.e., when the whole sample lies in the quasistatic regime. Nevertheless, these properties do not imply that the mechanical behavior in this regime is rate independent, as would be predicted by classical soil mechanics constitutive laws. First, Fig. 3(b) clearly shows that significant deformation rates exist within the quasistatic zones, both for $\Omega>\Omega_{t}$ and for $\Omega<\Omega_{t}$. When coexistence between inertial and quasistatic zones occurs (for $\Omega>\Omega_{t}$ ), the radial profiles of $I$ are actually completely continuous across the two zones. Hence, as already noted in [3], the quasistatic zones in the Couette cell appear to undergo a continuous creep 
which is incompatible with a rate-independent mechanical behavior. (With a rate-independent behavior, we would rather expect the material to remain immobile in the quasistatic zones, except in particular localization layers concentrating all the deformation [19].)

Second, even if there is no unique relationship between $\mu$ and $I$ for $I<I_{t}$, these two variables do nevertheless show clear correlations [Fig. 4(a)]. In particular, all data points obtained in quasistatic zones that coexist with an inertial zone (case $\Omega>\Omega_{t}$ ) appear to collapse on a master curve which smoothly connects with the $\mu(I)$ relationship obtained for $I>I_{t}$. For $\Omega<\Omega_{t}$, the data points follow distinct paths in the $\mu-I$ space according to the value of $\Omega$, but all these paths remain globally parallel to the master curve just described for $\Omega>\Omega_{t}$. Similar correlations are observed between $\Delta$ and $I$ in Fig. 4(b). In fact, the evolution of the fluctuation number $\Delta$ with $I$ in the quasistatic regime strongly resembles that of the friction coefficient $\mu$. Data points corresponding to $\Omega>\Omega_{t}$ collapse on a master curve, while data points obtained for $\Omega<\Omega_{t}$ follow distinct but approximately parallel paths.

These correlations and the similarity between the evolutions of $\mu$ and $\Delta$ with $I$ suggest the existence, in the quasistatic regime, of a unique relationship between these three quantities. To check this hypothesis, the three variables are represented in a three-dimensional (3D) plot in Fig. 5. Although not clearly evident on a planar representation, we observe that all the data points obtained in the quasistatic regime effectively appear to define a single surface in this plot. More quantitatively, a principal component analysis of the data set made of the triplets $\left[\log _{10}(I), \log _{10}(\Delta), \mu\right]$ has been performed. It shows that more than $99.9 \%$ of the data dispersion is explained by the two largest eigenvalues of the correlation matrix (Fig. 5). This constitutes a formal proof that the three variables are effectively linked by a unique relationship.

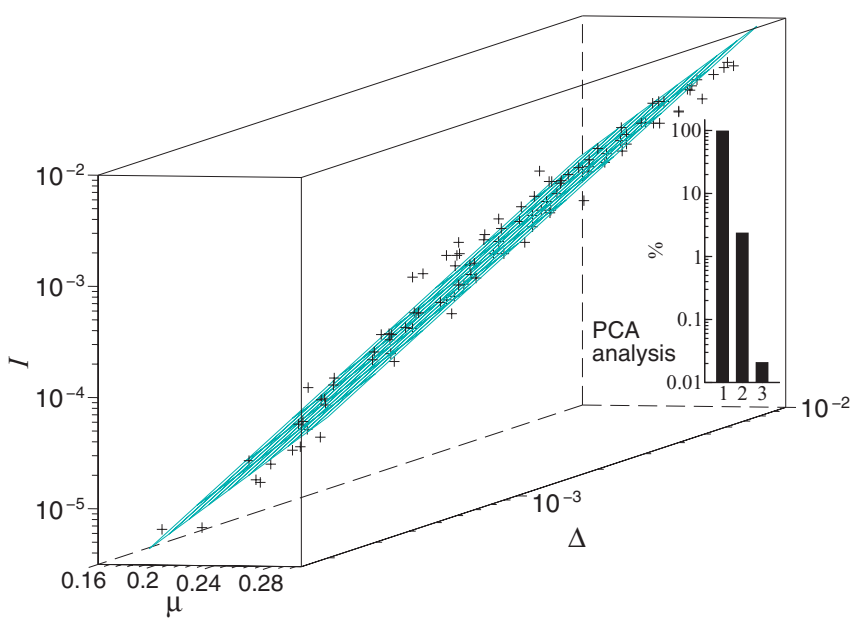

FIG. 5. (Color online) 3D plot representing the triplets constituted by the values of inertial number $I$ ( $\log _{10}$ scale), fluctuation number $\Delta\left(\log _{10}\right.$ scale $)$, and friction coefficient $\mu$ obtained in the quasistatic regime $\left(I<I_{t}\right)$. The histogram represents the three eigenvalues of the data-set correlation matrix inferred through principal value analysis (PCA). The surface plotted as an eyeguide corresponds to the plane defined by the two largest eigenvalues of the PCA.

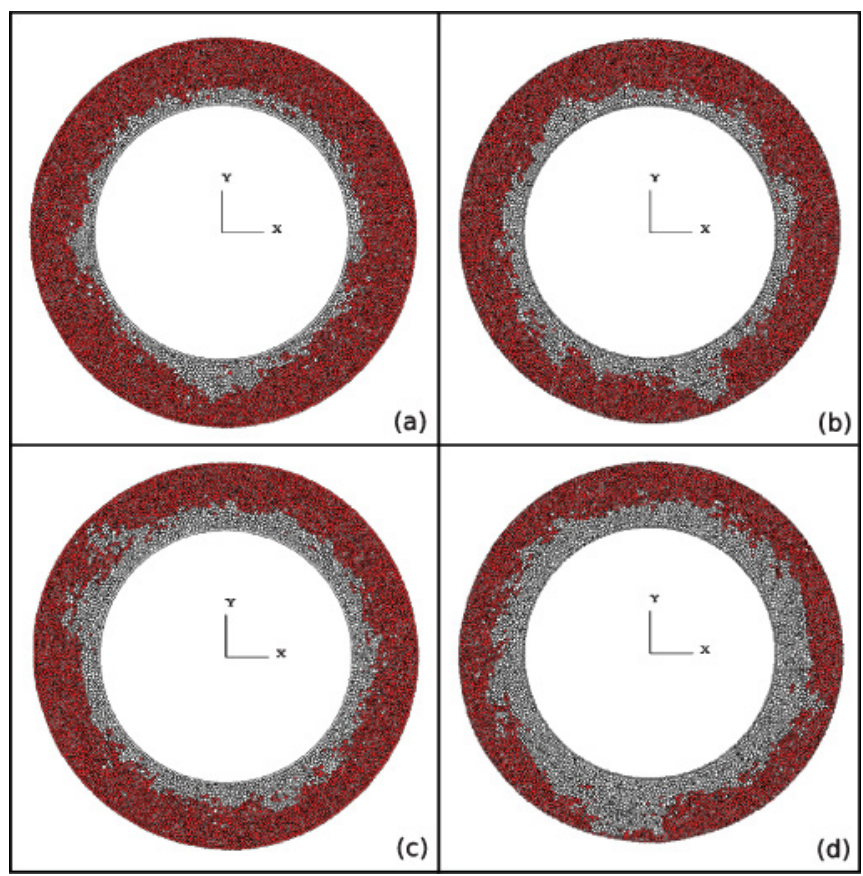

FIG. 6. (Color online) Instantaneous snapshots of particle velocities in the simulated Couette cell. White (light) particles are characterized by a velocity that exceeds a fixed threshold $\left(0.4 \mathrm{~m} \mathrm{~s}^{-1}\right.$ in this example), whereas red (dark) particles have a velocity below this threshold. (a) $\Omega=5$, (b) $\Omega=8$, (c) $\Omega=10$, and (d) $\Omega=20 \mathrm{rad} \mathrm{s}^{-1}$.

Hence, in spite of the absence of one-to-one relationships between $\mu$ and $I$ and $\Delta$ and $I$ in the quasistatic regime, these quantities are nevertheless strongly correlated through a three-variable relationship of the form $I=f(\mu, \Delta)$. Since it only involves local variables, this relationship, for which we shall propose an empirical expression in what follows, can be viewed as a local constitutive law characteristic of the quasistatic regime. Note, however, that the existence of this law does not imply that the rheology of the material is local. Indeed, and unlike in the inertial regime, the fluctuations in the quasistatic regime appear to be essentially produced by nonlocal processes. Qualitative observation of the particle velocities shows that fluctuations tend to organize into short-lived "bursts" that emerge at the boundary of the quasistatic zone (either at the inner cylinder or at the interface with the inertial zone) and then "propagate" into the material (Fig. 6). We also remark that when the fluctuation level at the boundary of a quasistatic zone is fixed, i.e., when the quasistatic zone coexists with an inertial zone, then the complete radial profile of the fluctuations inside this quasistatic zone is also fixed (cf. the collapse of the data points corresponding to $\Omega>\Omega_{t}$ in Fig. 4(b)]. These observations indicate that fluctuations in the quasistatic regime are in fact essentially sustained by the boundary conditions applied at the periphery of the quasistatic zones.

\section{EMPIRICAL CONSTITUTIVE RELATIONS}

\section{A. Derivation of a three-variable relationship}

From the principal component analysis presented above, it can be deduced that a linear function in terms of the 
variables $\log _{10}(I), \log _{10}(\Delta)$, and $\mu$ would constitute a good approximation for the three-variable constitutive relationship characterizing the quasistatic regime (at least for the values of $I, \mu$, and $\Delta$ covered in our simulations). However, an even better empirical expression for this relationship can be derived by analyzing the dependence between $I$ and $\Delta$ at constant values of $\mu$. Such an analysis is possible for $\Omega<\Omega_{t}$, i.e., when the whole sample lies in the quasistatic regime, owing to the fact that the friction coefficient $\mu$ is independent of $\Omega$ in this case. Therefore, studying the dependence between $I$ and $\Delta$ for data determined at constant values of radius $r$ is equivalent to considering constant values of $\mu$.

Figure 7(a) shows that for each value of friction coefficient $\mu$, the evolution of the fluctuation number $\Delta$ as a function of the inertial number $I$ approximately follows a power-law of the form

$$
\Delta=C_{\mathrm{QS}} I^{\beta(\mu)}
$$

with a prefactor $C_{\mathrm{QS}} \approx 0.59$ independent of $\mu$, and an exponent $\beta$ increasing with $\mu$. Furthermore, the dependence of this exponent $\beta$ with $\mu$ appears to be essentially linear [Fig. 7(b)]:

$$
\beta(\mu)=\beta_{0}+\beta_{1} \mu,
$$

with $\beta_{0} \approx 0.29$ and $\beta_{1} \approx 1.80$. Quantitatively, in terms of least-squares error, these empirical expressions (3) and (4) provide a fit to the surface defined by the quasistatic data points in Fig. 5 which is $20 \%$ better than the fit obtained with the linear relationship derived from principal component analysis.

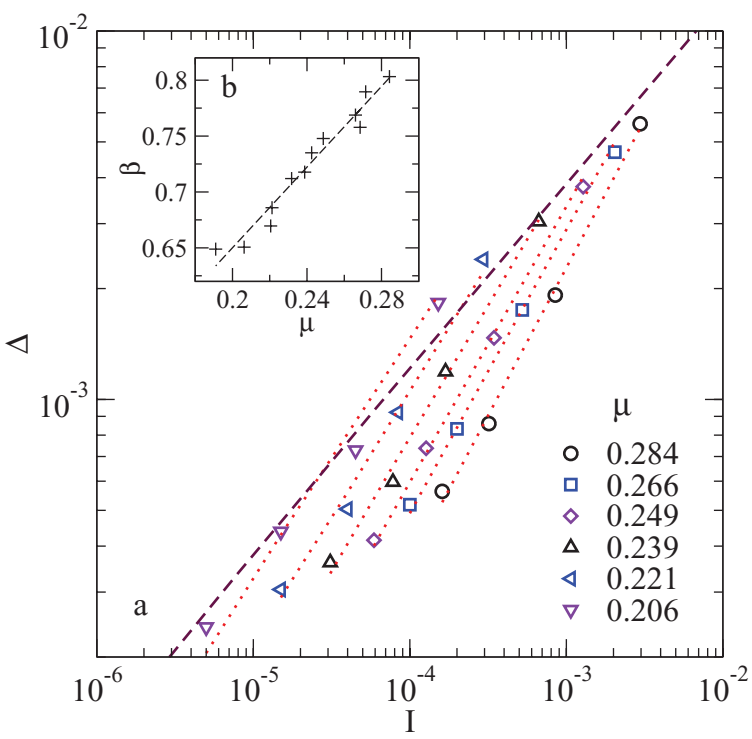

FIG. 7. (Color online) (a) Fluctuation number $\Delta$ as a function of inertial number $I$ at fixed values of friction coefficient $\mu$ (see legend). The represented data come from simulations in which the whole sample lies in the quasistatic regime $\left(\Omega<\Omega_{t}\right)$. Dotted lines correspond to the best power law fits Eq. (3) obtained for each value of $\mu$. The dashed line represents the extrapolation of the relationship between $\Delta$ and $I$ established in inertial regime Eq. (2). (b) Evolution of power-law exponent $\beta$ with friction coefficient $\mu$. The dashed line represents the best linear fit Eq. (4).

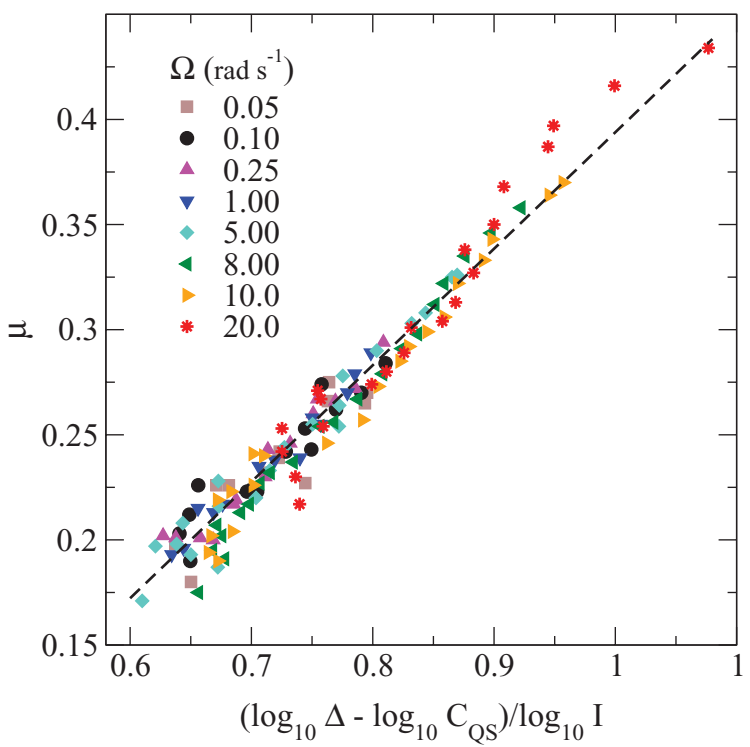

FIG. 8. (Color online) Friction coefficient $\mu$ as a function of the composite variable $\left(\log _{10} \Delta-\log _{10} C_{\mathrm{QS}}\right) / \log _{10} I$. All data points determined from the simulations are represented, the different symbols referring to the values of imposed rotation velocity $\Omega$. The dashed line represents the prediction of Eq. (5).

Equations (3) and (4) can be rewritten in a more compact form as

$$
\mu=\frac{1}{\beta_{1}}\left(\frac{\log _{10} \Delta-\log _{10} C_{\mathrm{QS}}}{\log _{10} I}-\beta_{0}\right) .
$$

As a further validation of this expression, Fig. 8 shows that all data points determined in the quasistatic regime, including those corresponding to $\Omega>\Omega_{t}$ which were not taken into account in the establishment of Eqs. (3) and (4), effectively collapse on a single master curve when plotted in terms of $\mu$ versus $\left(\log _{10} \Delta-\log _{10} C_{\mathrm{QS}}\right) / \log _{10} I$. Hence, we argue that Eq. (5), or equivalently Eqs. (3) and (4), can be regarded as good approximations of the three-variable constitutive relationship characterizing the quasistatic regime. We emphasize, however, that these equations are purely empirical at this stage, and that we cannot rule out the possible existence of alternative expressions that would produce an even better fit to the data.

\section{B. Extrapolation to the inertial regime}

An important point not mentioned above is that Fig. 8 also includes the data determined in the inertial regime. Interestingly, these data appear to collapse on the same master curve as those corresponding to the quasistatic regime. This unexpected result seems to indicate that Eq. (5), established in the quasistatic regime, would also remain valid in the inertial regime. To check this prediction, Eq. (5) can be combined with Eq. (2) relating $\Delta$ and $I$ in the inertial regime, to yield the following relationship between the variables $\mu$ and $I$ alone:

$$
\mu=\mu_{0}-\frac{\mu_{1}}{\log _{10} I},
$$

with $\mu_{0}=\left(1 / 2-\beta_{0}\right) / \beta_{1} \approx 0.12$ and $\mu_{1}=\left(\log _{10} C_{\mathrm{QS}}-\right.$ $\left.\log _{10} C_{I}\right) / \beta_{1} \approx 0.38$. As shown in Fig. 9, this expression 


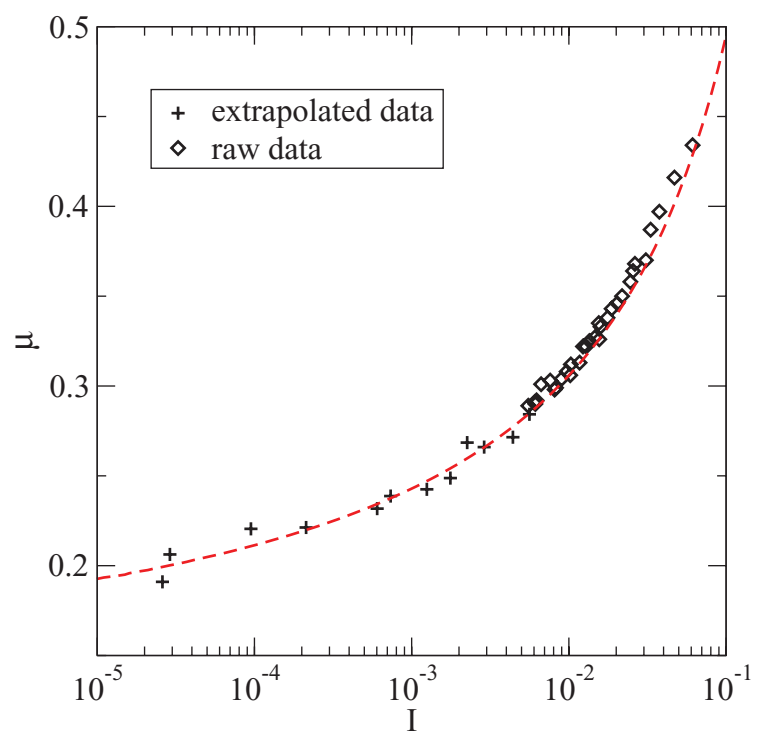

FIG. 9. (Color online) Relationship between friction coefficient $\mu$ and inertial number $I$ for the data points obtained in the inertial regime $\left(I>I_{t}\right)$ and for the data points extrapolated from the quasistatic regime (see text). The dashed curve represents Eq. (6).

effectively provides an excellent fit to the $\mu(I)$ relationship observed in the inertial regime, with fewer parameters than Eq. (1) used previously [see also Fig. 4(a)]. Furthermore, we emphasize that in this case, the two parameters $\mu_{0}$ and $\mu_{1}$ were not adjusted on the inertial data, but directly derive from Eqs. (2) and (5), the latter having been established using quasistatic data only.

Hence, the three-variable constitutive law between $I, \mu$, and $\Delta$ characteristic of the quasistatic regime turns out to be also valid in the inertial regime. This three-variable law actually includes the $\mu(I)$ relationship characterizing the inertial regime, which emerges as soon as fluctuations begin to be governed by the local law (2). In consequence, a virtual continuation in the quasistatic regime of the inertial $\mu(I)$ relationship can be drawn assuming that Eq. (2) remains valid for $I<I_{t}$. This continuation is shown in Fig. 9 using directly Eq. (6), and through extrapolated data points which have been computed from quasistatic results as the intersects, for each value of friction coefficient $\mu$, between relationships (3) and (2) (see Fig. 7). Note that the value of the exponent $\beta$ used to compute these intersects was the best-fitting value obtained for each friction coefficient, and not the linear approximation given by Eq. (4). It is interesting to note that these extrapolated data points figure in the exact continuity of the data points obtained in the inertial regime, which constitutes further evidence that the quasistatic and inertial regimes are effectively governed by the same underlying constitutive law.

\section{DISCUSSION AND CONCLUSIONS}

The analysis presented in this paper sheds new light on the rheological behavior of granular materials in the quasistatic regime. Our results clearly show that this regime is characterized both by a friction coefficient independent of the shear rate, and by a local, three-variable constitutive relationship between the inertial number, the friction coefficient, and the normalized velocity fluctuations. Importantly, this constitutive law directly relates the occurrence of creep in quasistatic zones to the existence of fluctuations. It is only in the absence of fluctuations that the material is jammed and $I=0$ for whatever the level of stress. As soon as fluctuations exist, the material can flow even under very small applied stresses, with an apparent viscosity that is a direct function of the fluctuation amplitude [see Eq. (3)]. The key role played by the fluctuations in the rheology of quasistatic granular materials has already been hypothesized by several authors [13,15,22,23]. Our study thus formally demonstrates this assumption by showing, directly from local mechanical data, that fluctuations must be accounted for in the constitutive relationship. An interesting perspective for future work would now be to go beyond the purely empirical approach presented here, and develop a theoretical framework capable of yielding a three-variable constitutive law compatible with our results.

Another important outcome of this study is the fact that the three-variable constitutive law obtained in the quasistatic regime appears to be also valid in the inertial regime. When combined with the expression governing the fluctuations in the inertial regime Eq. (2), this constitutive law yields a one-to-one $\mu(I)$ relationship which, though of a different form from the relationships previously proposed in the literature $[2,4]$, provides an excellent fit to the inertial data. Hence, the distinction between the quasistatic and inertial regimes cannot be related to the rate-dependent or rate-independent character of the mechanical behavior. Both regimes are in fact characterized by the same underlying constitutive law involving the variables $I, \mu$, and $\Delta$.

In spite of this similarity in mechanical behavior, the quasistatic and inertial regimes are nevertheless separated by an abrupt transition at $I=I_{t}$. Elaborating from our results, we propose that the "true" origin of this transition is in fact related to the mode of production of the fluctuations within the material. As already pointed by other workers [1,2], in the inertial regime the fluctuations necessary for the flow are created locally by the flow itself. This is the meaning of Eq. (2), and explains the possibility of reducing the three-variable constitutive law to a local relationship between $\mu$ and $I$ alone. On the contrary, in the quasistatic regime, the fluctuations result from nonlocal processes [9-11] and, as shown by our results, are essentially sustained by sources localized at the boundaries of the quasistatic zones (either at the wall or at the interface with the neighboring inertial zone). In particular, when a quasistatic zone coexists with an inertial one, the fluctuations within the former and, as a consequence, its apparent rheology (namely, the apparent relationship between $\mu$ and $I$ ), are completely controlled by the latter. In the absence of boundary sources, on the contrary, the fluctuations in the bulk would rapidly die off, and $I$ would tend to 0 . Eventually, the three-variable relationship presented in this paper will thus need to be complemented by a nonlocal evolution equation for the fluctuations in quasistatic regime.

As a conclusion, the quasistatic-to-inertial transition in granular materials thus appears to correspond to a transition between a regime where the fluctuations are governed by nonlocal processes and a regime where fluctuations are produced locally. This transition occurs at a critical value of 
$I$ or equivalently [according to Eq. (2)], at a critical value of $\Delta, \Delta_{t} \approx 8 \times 10^{-3}$ in our case, which can be interpreted as the fluctuation level above which the mechanisms for long-range propagations of the fluctuations become inefficient. Following this interpretation, the parameters $\Delta_{t}$ and $I_{t}$ should thus represent intrinsic characteristics of the material, whose value should be independent of the considered system. This prediction tends to be confirmed by the results reported in $[2,3]$, in which different system sizes and system geometries result to apparently constant values of $I$ at the quasistatic-to-inertial transition. Yet, the uncertainties associated with the determination of $I_{t}$ and the different methods employed to define this parameter (from global or local measurements) render difficult the comparisons among existing studies; further work would be needed to conclude on the intrinsic nature of $I_{t}$.

\section{ACKNOWLEDGMENT}

Financial support from the French National Agency for Research is acknowledged (ANR project MONHA).
[1] G. D. R. MiDi, Eur. Phys. J. E 14, 341 (2004).

[2] F. da Cruz, S. Emam, M. Prochnow, J. N. Roux, and F. Chevoir, Phys. Rev. E 72, 021309 (2005).

[3] G. Koval, J. N. Roux, A. Corfdir, and F. Chevoir, Phys. Rev. E 79, 021306 (2009).

[4] P. Jop, Y. Forterre, and O. Pouliquen, Nature 441, 727 (2006).

[5] Y. Forterre and O. Pouliquen, Annu. Rev. Fluid Mech. 40, 1 (2008).

[6] J. N. Roux and G. Combe, C.R. Phys. 3, 131 (2002).

[7] T. S. Komatsu, S. Inagaki, N. Nakagawa, and S. Nasuno, Phys. Rev. Lett. 86, 1757 (2001).

[8] P. Jop, Y. Forterre, and O. Pouliquen, Phys. Fluids 19, 088102 (2007).

[9] F. Radjai and S. Roux, Phys. Rev. Lett. 89, 064302 (2002).

[10] G. Chambon, J. Schmittbuhl, A. Corfdir, J.-P. Vilotte, and S. Roux, Phys. Rev. E 68, 011304 (2003).

[11] O. Pouliquen, Phys. Rev. Lett. 93, 248001 (2004).

[12] P. Mills, P. G. Rognon, and F. Chevoir, Eur. Phys. Lett. 81, 64005 (2008).

[13] O. Pouliquen and Y. Forterre, Phil. Trans. Math. Phys. Eng. Sci. 367, 5091 (2009).
[14] L. Bocquet, W. Losert, D. Schalk, T. C. Lubensky, and J. P. Gollub, Phys. Rev. E 65, 011307 (2001).

[15] G. I. Tardos, S. McNamara, and I. Talu, Powder Technol. 131, 23 (2003).

[16] F. Radjai and F. Dubois, Discrete-Element Modeling of Granular Materials (ISTE, London, and Wiley, New York, 2011).

[17] C. T. Veje, D. W. Howell, and R. P. Behringer, Phys. Rev. E 59, 739 (1999).

[18] D. M. Mueth, G. F. Debregeas, G. S. Karczmar, P. J. Eng, S. R. Nagel, and H. M. Jaeger, Nature 406, 385 (2000).

[19] A. Zervos, I. Vardoulakis, M. Jean, and P. Lerat, Mech. Cohes. Frict. Mat. 5, 305 (2000)

[20] P. A. Cundall and O. D. L. Strack, Géotechnique 29, 47 (1979).

[21] B. Cambou and M. Jean, Micromécanique des Matériaux Granulaires, Traité MIM, Série Géomatériaux (Hermes Science, London, 2001).

[22] L. Bocquet, J. Errami, and T. C. Lubensky, Phys. Rev. Lett. 89, 184301 (2002).

[23] K. A. Reddy, Y. Forterre, and O. Pouliquen, Phys. Rev. Lett. 106, 108301 (2011). 